\title{
RIGHT ALTERNATIVE ALGEBRAS AND WEDDERBURN PRINCIPAL THEOREM
}

\begin{abstract}
ARMIN THEDY
ABSTRACT. Examples are given of right alternative algebras and of quadratic Jordan algebras (the latter only over fields of characteristic two) without a Wedderburn splitting but such that at least one $u$-isotope admits a Wedderburn splitting. An example of a noncommutative Jordan algebra over a field of characteristic $\neq 2$ is given such that no $u$-isotope admits a Wedderburn splitting.
\end{abstract}

Everyone who has worked with right alternative algebras knows that the defining relations are rather weak. Hence there should exist a lot of right alternative algebras. But only a few examples may be found scattered in the literature. In this note we study a family of unital right alternative $k$-algebras of rank 8 by giving the multiplication table of a basis that depends on 5 independent parameters. Each of our right alternative $k$-algebras $R$ has a radical $Z$ of rank 4 such that $Z Z=0$ and $R / Z$ is isomorphic to the full 2-rowed matrix ring over $k$. We determine for which values of the parameters our algebras admit a direct splitting $R=S \oplus Z$, where $S$ is a subalgebra of $R$ isomorphic to $R / Z$. As a consequence one sees that the Wedderburn Principal Theorem does not hold in the class of all right alternative algebras. But $R$ has at least one $u$-isotope admitting a Wedderburn splitting. Moreover we get an example of a quadratic Jordan algebra $R^{+}$of characteristic two that has no Wedderburn splitting. This shows that the Wedderburn Principal $=$ Albert-Penico-Taft Theorem for linear Jordan algebras cannot be extended to quadratic Jordan algebras of characteristic two. But there is a $u$-isotope admitting a Wedderburn splitting. In a final section we present a noncommutative Jordan algebra with the property that no $u$-isotope admits a Wedderburn splitting.

Right alternative algebras. Let $k$ be a commutative associative ring with unit element. Choose as parameters elements ' $\alpha, \varepsilon, \tau, \xi, \kappa$ of $k$ with the only restriction that $\kappa$ is invertible. Let $R$ be a free $k$-module of rank 8 with basis denoted by $c_{1}, c_{2}, c_{12}, c_{21}, z_{1}, z_{2}, z_{12}, z_{21}$. We give $R$ the structure of a $k$ algebra $R=R(\alpha, \varepsilon, \tau, \xi, \kappa)$ by requiring that these elements multiply according to the following multiplication table.

Received by the editors November 14, 1977.

AMS (MOS) subject classifications (1970). Primary 17A30, 17C50, 17A15, 17C10.

Key words and phrases. Wedderburn Principal Theorem, Albert-Penico-Taft Theorem, right alternative algebras, quadratic Jordan algebras, noncommutative Jordan algebras, $u$-isotope.

c) American Mathematical Society 1978 


\begin{tabular}{|c|c|c|c|c|}
\hline & $c_{1}$ & $c_{2}$ & $c_{12}$ & $c_{21}$ \\
\hline$c_{1}$ & $c_{1}$ & 0 & $c_{12}$ & $z_{2}$ \\
\hline$c_{2}$ & 0 & $c_{2}$ & 0 & $c_{21}-z_{2}$ \\
\hline$c_{12}$ & 0 & $c_{12}$ & $\epsilon z_{1}$ & $c_{1}+\alpha z_{1}-z_{21}$ \\
\hline$c_{21}$ & $c_{21}-z_{2}$ & $z_{2}$ & $c_{2}+\epsilon \kappa z_{2}+z_{21}+\tau z_{1}$ & $\kappa(\alpha+\tau) z_{2}$ \\
\hline$z_{1}$ & $z_{1}$ & 0 & 0 & $z_{12}-\kappa \tau z_{1}$ \\
\hline$z_{2}$ & 0 & $z_{2}$ & $z_{21}+\tau z_{1}$ & 0 \\
\hline$z_{12}$ & $\kappa \tau z_{1}$ & $z_{12}-\kappa \tau z_{1}$ & $z_{1}$ & $\kappa \tau\left(z_{12}-\kappa \tau z_{1}\right)$ \\
\hline \multirow[t]{2}{*}{$z_{21}$} & $z_{21}$ & 0 & 0 & $z_{2}-\tau\left(z_{12}-\kappa \tau z_{1}\right)$ \\
\hline & $z_{1}$ & $z_{2}$ & $z_{12}$ & $z_{21}$ \\
\hline$c_{1}$ & $z_{1}$ & 0 & $z_{12}-\kappa \tau z_{1}$ & 0 \\
\hline$c_{2}$ & 0 & $z_{2}$ & $\kappa \tau z_{1}$ & $z_{21}$ \\
\hline$c_{12}$ & 0 & $\kappa^{-1} z_{12}-\tau z_{1}$ & $-\xi z_{1}$ & $\kappa^{-1}(1+\xi) z_{1}$ \\
\hline$c_{21}$ & $\kappa\left(\tau z_{1}+z_{21}\right)$ & 0 & $-\kappa \tau\left(z_{12}-\kappa \tau z_{1}\right)+\kappa(1-\xi) z_{2}$ & $\tau\left(z_{12}-\kappa \tau z_{1}\right)+\xi z_{2}$ \\
\hline$z_{1}$ & 0 & 0 & 0 & 0 \\
\hline$z_{2}$ & 0 & 0 & 0 & 0 \\
\hline$z_{12}$ & 0 & 0 & 0 & 0 \\
\hline$z_{21}$ & 0 & 0 & 0 & 0 \\
\hline
\end{tabular}

MULTiplication TABLE

Since $k$ is arbitrary the algebra $R$ is right alternative in case $(x y) y=x y^{2}$ and $(x y) y^{2}=x y^{3}$ holds for all $x, y$ in $R$ [12]. Since $c_{1}+c_{2}$ is a unit element of $R$ it suffices to prove these equations for $x \in\left\{c_{1}, c_{12}, c_{21}, z_{1}, z_{2}, z_{12}, z_{21}\right\}$ and all $y=\eta_{1} c_{1}+\eta_{2} c_{2}+\eta_{12} c_{12}+\eta_{21} c_{21}+\zeta_{1} z_{1}+\zeta_{2} z_{2}+\zeta_{12} z_{12}+\zeta_{21} z_{21} \in$ $R, \eta_{2}=0$. One has

$$
\begin{aligned}
y^{2}= & \left(\eta_{1}+\eta_{2}\right) y+\left(\eta_{12} \eta_{21}-\eta_{1} \eta_{2}\right)\left(c_{1}+c_{2}\right) \\
& +\beta_{1} z_{1}+\beta_{2} z_{2}+\beta_{12}\left(z_{12}+\kappa z_{21}\right)
\end{aligned}
$$

with

$$
\begin{aligned}
\beta_{1}:= & \eta_{12}^{2} \varepsilon+\left(\eta_{1}-\eta_{2}\right) \zeta_{1}+\eta_{12} \eta_{21}(\alpha+\tau) \\
& +\eta_{12} \zeta_{12}(1-\xi)+\eta_{12} \zeta_{21}(1+\xi) \kappa^{-1}, \\
\beta_{2}:= & \eta_{12} \eta_{21} \varepsilon \kappa-\left(\eta_{1}-\eta_{2}\right) \zeta_{2}+\eta_{21}^{2}(\alpha+\tau) \kappa \\
& +\eta_{21} \zeta_{12}(1-\xi) \kappa+\eta_{21} \zeta_{21}(1+\xi), \\
\beta_{12}:= & \eta_{12} \zeta_{2} \kappa^{-1}+\eta_{21} \zeta_{1}
\end{aligned}
$$

satisfying the equation $\eta_{12} \beta_{2}-\eta_{21} \beta_{1} \kappa+\left(\eta_{1}-\eta_{2}\right) \beta_{12} \kappa=0$. With these 
remarks the reader will easily verify $(x y) y=x y^{2}$ and $(x y) y^{2}=x y^{3}$. Hence for all choices of $\alpha, \varepsilon, \tau, \xi, \kappa R$ is a right alternative algebra.

Trivially $Z:=k z_{1}+k z_{2}+k z_{12}+k z_{21}$ is an ideal of $R$ such that $Z Z=0$ and $R / Z$ is the 2-rowed matrix ring over $k$. Each element of $Z$ is an absolute zero divisor of $R$ and $R / Z$ has no absolute zero divisor besides zero. Hence $Z$ is the (lower) radical of $R$ [2], [13], [14], [15].

The Wedderburn Principal Theorem holds for $R$ if and only if $R$ contains a subalgebra $S$ such that $R=S \oplus Z$. The canonical epimorphism of $R$ onto $R / Z$ restricts to an isomorphism $\varphi$ of $S$ onto $R / Z$. Hence the Wedderburn Principal Theorem holds for $R$ if and only if $R$ contains elements $d_{1}, d_{2}, d_{12}, d_{21}$ such that $d_{1}+d_{2}=c_{1}+c_{2}, \varphi\left(d_{1}\right)=c_{1}+Z, \varphi\left(d_{2}\right)=c_{2}+Z$, $\varphi\left(d_{12}\right)=c_{12}+Z, \varphi\left(d_{21}\right)=c_{21}+Z$ and $d_{1}^{2}=d_{1}, d_{2}^{2}=d_{2}, d_{1} d_{12}=d_{12} d_{2}=$ $d_{12}, d_{21} d_{1}=d_{2} d_{21}=d_{21}, d_{12} d_{21}=d_{1}, d_{21} d_{12}=d_{2}, d_{12}^{2}=0=d_{21}^{2}$.

The only orthogonal idempotents $d_{1}, d_{2}$ of $R$ satisfying $\varphi\left(d_{1}\right)=c_{1}+Z$, $\varphi\left(d_{2}\right)=c_{2}+Z$ and $d_{1}+d_{2}=c_{1}+c_{2}$ are $d_{1}=c_{1}+\mu z_{12}-\nu z_{21}$ and $d_{2}=c_{2}$ $-\mu z_{12}+\nu z_{21}$ with $\mu, \nu \in k$. The Albert (= Peirce)-decomposition $R=R_{11}$ $+R_{12}+R_{21}+R_{22}$ with respect to the complete orthogonal system of idempotents $d_{1}, d_{2}$ is given by

$$
\begin{aligned}
& R_{11}=k d_{1}+k z_{1}, \\
& R_{12}=k\left(c_{12}-\mu(1-\xi) z_{1}+\nu(1+\xi) \kappa^{-1} z_{1}\right)+k z_{12}, \\
& R_{21}=k\left(c_{21}+\mu(1-\xi) \kappa z_{2}-\nu(1+\xi) z_{2}-(\mu \kappa+\nu) \tau z_{12}\right)+k z_{21}, \\
& R_{22}=k d_{2}+k z_{2}[13],[14],[15] .
\end{aligned}
$$

Hence the only candidates for $d_{12}, d_{21}$ are

$$
\begin{aligned}
& d_{12}:=c_{12}-\mu(1-\xi) z_{1}+\nu(1+\xi) \kappa^{-1} z_{1}+\rho z_{12} \text { and } \\
& d_{21}:=c_{21}+\mu(1-\xi) \kappa z_{2}-\nu(1+\xi) z_{2}-(\mu \kappa+\nu) \tau z_{12}+\sigma z_{21} \text { with } \rho, \sigma \in k \text {. } \\
& \text { We calculate } \\
& \begin{array}{ll}
d_{12} d_{1}=(\rho \kappa \tau-\mu) z_{1}, & d_{1} d_{21}=(1-\nu) z_{2}, \\
d_{1} d_{12}+d_{12} d_{1}=d_{12}, & d_{1} d_{21}+d_{21} d_{1}=d_{21},
\end{array} \\
& d_{12} d_{21}=d_{1}+(\rho \kappa \tau-\mu) z_{12}-(1-\nu) z_{21}+\left(\alpha+\tau+\sigma \kappa^{-1}(1+\xi)\right. \\
& +(\mu \kappa+\nu) \tau(\xi-1)+\kappa \tau(\mu-\rho \kappa \tau)+\tau(\nu-1)) z_{1}, \\
& d_{21} d_{12}=d_{2}-(\rho \kappa \tau-\mu) z_{12}+(1-\nu) z_{21}+\kappa(\varepsilon+\rho(1-\xi)) z_{2} \\
& +(\tau(1-\nu)+\kappa \tau(\rho \kappa \tau-\mu)) z_{1} \text {, } \\
& d_{21}^{2}=(\varepsilon+\rho(1-\xi)) z_{1} \text {, } \\
& d_{21}^{2}=\kappa\left(\alpha+\tau+\sigma \kappa^{-1}(1+\xi)+(\mu \kappa+\nu) \tau(\xi-1)\right) z_{2} .
\end{aligned}
$$

Hence $R$ allows a Wedderburn splitting if and only if there exist $\mu, \nu, \rho, \sigma \in$ $k$ such that the following equations hold:

$$
\begin{aligned}
& \nu=1, \\
& \mu=\rho \kappa \tau, \\
& \rho(\xi-1)=\varepsilon, \\
& \sigma(\xi+1)=-\kappa\left(\alpha+\tau \xi+\varepsilon \kappa^{2} \tau^{2}\right) .
\end{aligned}
$$


From this one concludes

THEOREM 1. Let $k$ be a field. Then $R=R(\alpha, \varepsilon, \tau, \xi, \kappa)$ does not admit $a$ Wedderburn splitting if and only if $\xi=1, \varepsilon \neq 0$ or $\xi=-1, \alpha \neq \tau-\varepsilon \kappa^{2} \tau^{2}$.

Especially for $\kappa=\varepsilon=\xi=1, \alpha=\tau=0$ one obtains a right alternative algebra for which the Wedderburn Principal Theorem fails to hold. On the other hand for alternative algebras and some more general classes of algebras the Wedderburn Principal Theorem is true [1], [4]-[11].

Quadratic Jordan algebras. Every right alternative algebra $R$ carries the structure of a quadratic Jordan algebra $R^{+}$with $U$-operator $U_{x}(y)=(x y) x$ [2], [12], [13].

If 2 is an invertible element of the ground ring $k$, then the concepts quadratic Jordan algebras and linear Jordan algebras are equivalent [2], [12]. For finite-dimensional (linear) Jordan algebras over a field $k$ of characteristic $\neq 2$ the Wedderburn Principal $=$ Albert-Penico-Taft Theorem holds [2], [11]. In [11] a 4-dimensional example of a noncommutative Jordan algebra without a Wedderburn splitting was given. In case of characteristic two this example is a linear Jordan algebra not admitting a Wedderburn splitting. But in 1970 McCrimmon observed in a discussion with the author that this example admits a Wedderburn splitting as quadratic Jordan algebra since the radical has dimension three. The problem whether Jordan algebras of characteristic two admit Wedderburn splittings has resisted all efforts for many years. Now we can decide it by giving a counterexample.

THEOREM 2. If $k$ is a field of characteristic two then the quadratic Jordan algebra $R^{+}$does not admit a Wedderburn splitting if and only if $\xi=1, \varepsilon \neq 0$ or $\xi=1, \alpha \neq \tau$.

Proof. The radical of $R^{+}$is $Z$ since $R^{+} / Z \cong M_{2}(k)^{+}$is a simple quadratic Jordan algebra. If there exists a Jordan subalgebra $S^{+}$of $R^{+}$such that $R^{+}=S^{+} \oplus Z$, then there are $\mu, \nu, \rho, \sigma, \chi, \omega \in k$ such that $d_{1}:=c_{1}+$ $\mu z_{12}-\nu z_{21}, d_{2}:=c_{2}-\mu z_{12}+\nu z_{21}$ are idempotents in $S^{+}$and $d_{12}:=c_{12}+$ $\rho z_{12}+\sigma z_{21}, d_{21}:=c_{21}+\chi z_{12}+\omega z_{21}$ are elements of $S^{+}$such that $d_{12} \circ d_{21}$ $=c_{1}+c_{2}$. But

$$
\begin{aligned}
d_{12} \circ d_{21}= & c_{1}+c_{2}+\left(\alpha+\tau+\chi(1-\xi)+\omega \kappa^{-1}(1+\xi)\right) z_{1} \\
& +(\varepsilon \kappa+\rho \kappa(1-\xi)+\sigma(1+\xi)) z_{2} \\
\neq & c_{1}+c_{2}
\end{aligned}
$$

for $\xi=1, \varepsilon \neq 0$ or $\xi=1, \alpha \neq \tau$. The converse follows from Theorem 1 .

Isotopes of $R$. If $R$ is a unital right alternative algebra and $u \in R$ is invertible, then the $k$-module $R$ under the new multiplication $x_{u} \cdot y:=(x u) y$ is a right alternative algebra $R^{(u)}$ called the $u$-isotope of $R$ [12]. An algebra $S$ isomorphic to an isotope of $R$ is called isotopic to $R$. Since isotopy is an 
equivalence relation more general than isomorphy one may ask whether for each right alternative algebra an isotope can be found admitting a Wedderburn splitting. As a first step in this direction we study the isotopes of our special class of right alternative algebras $R(\alpha, \varepsilon, \tau, \xi, \kappa)$.

An element $u \in R$ is invertible if and only if $u+Z \in R / Z$ is invertible. Hence

$$
u=\lambda_{1} c_{1}+\lambda_{2} c_{2}+\lambda_{12} c_{12}+\lambda_{21} c_{21}+\rho_{1} z_{1}+\rho_{2} z_{2}+\rho_{12} z_{12}+\rho_{21} z_{21}
$$

is invertible if and only if $\lambda_{1} \lambda_{2}-\lambda_{12} \lambda_{21} \neq 0$. Without loss of generality we may assume $\lambda_{1} \lambda_{2}-\lambda_{12} \lambda_{21}=1$ (for invertible $\lambda \in k$ one has $R^{(\lambda u)} \simeq R^{(u)}$ ). Then the inverse $u^{-1}$ of $u$ equals

$$
u^{-1}=\lambda_{2} c_{1}+\lambda_{1} c_{2}-\lambda_{12} c_{12}-\lambda_{21} c_{21}+\sigma_{1} z_{1}+\sigma_{2} z_{2}+\sigma_{12} z_{12}+\sigma_{21} z_{21}
$$

with

$$
\begin{aligned}
\sigma_{1} & =\beta \lambda_{12} \lambda_{2}-\lambda_{2}^{2} \rho_{1}-\lambda_{12}^{2} \rho_{2} \kappa^{-1} \\
\sigma_{2} & =\beta \lambda_{21} \lambda_{1} \kappa-\lambda_{1}^{2} \rho_{2}-\lambda_{21}^{2} \rho_{1} \kappa, \\
\sigma_{12} & =-\rho_{12}-\beta \lambda_{12} \lambda_{21}+\lambda_{2} \lambda_{21} \rho_{1}+\lambda_{1} \lambda_{12} \rho_{2} \kappa^{-1}, \\
\sigma_{21} & =-\rho_{21}-\beta \lambda_{12} \lambda_{21} \kappa+\lambda_{1} \lambda_{12} \rho_{2}+\lambda_{2} \lambda_{21} \rho_{1} \kappa
\end{aligned}
$$

and $\beta$ given by $\beta=(\alpha+\tau) \lambda_{21}+\varepsilon \lambda_{12}+\rho_{12}(1-\xi)+\rho_{21} \kappa^{-1}(1+\xi)$. The algebra $R^{(u)}$ is again unital with unit element $u^{-1}$. The radical of $R^{(u)}$ equals $Z$ and satisfies $Z_{u} \cdot Z=0$. The factor algebra $R^{(u)} / Z=(R / Z)^{(u+Z)}$ is isomorphic to $M_{2}(k)$. This leads us to consider for fixed $\mu, \nu, \rho, \sigma \in k$ the following elements of $R$

$$
\begin{aligned}
d_{1}:= & \lambda_{2} c_{1}-\lambda_{12} c_{12}+\sigma_{1} z_{1}+\left(\sigma_{12}+\mu\right) z_{12}-\nu z_{21}, \\
d_{2}:= & \lambda_{1} c_{2}-\lambda_{21} c_{21}+\sigma_{2} z_{2}+\left(\sigma_{21}+\nu\right) z_{21}-\mu z_{12}, \\
d_{12}:= & \lambda_{1} c_{12}-\lambda_{21} c_{1}+\left(\left(\sigma_{21}+\nu\right)(1+\xi) \kappa^{-1}-\lambda_{21}(\alpha+\tau)-\mu(1-\xi)\right) z_{1} \\
& +\lambda_{21} z_{21}+\rho z_{12} \\
d_{21}:= & \lambda_{2} c_{21}-\lambda_{12} c_{2}+\left(\left(\sigma_{12}+\mu\right)(1-\xi) \kappa-\lambda_{12} \varepsilon \kappa-\nu(1+\xi)\right) z_{2} \\
& -\left(\sigma_{12} \kappa+\mu \kappa+\nu\right) \tau z_{12}+\sigma z_{21} .
\end{aligned}
$$

After calculating

$$
\begin{aligned}
d_{1} u= & c_{1}+\left(\lambda_{12} \lambda_{21} \tau-\lambda_{1} \rho_{12} \kappa \tau+\lambda_{21} \rho_{1} \kappa \tau+\mu\left(\lambda_{1} \kappa \tau+\lambda_{12}\right)-\sigma_{2} \lambda_{12} \tau\right) z_{1} \\
+ & \left(\lambda_{2} \lambda_{21}-\nu \lambda_{21}\right) z_{2}+\left(\lambda_{21} \rho_{21} \tau-\lambda_{21} \rho_{12} \kappa \tau+\left(\sigma_{21}+\nu\right) \lambda_{21} \tau\right. \\
& \left.+\mu\left(\lambda_{2}+\lambda_{21} \kappa \tau\right)\right) \\
& \cdot\left(z_{12}-\kappa \tau z_{1}\right)+\left(\lambda_{12} \lambda_{21}-\nu \lambda_{1}\right) z_{21},
\end{aligned}
$$




$$
\begin{gathered}
d_{2} u=c_{2}-\left(\lambda_{12} \lambda_{21} \tau-\lambda_{1} \rho_{12} \kappa \tau+\lambda_{21} \rho_{1} \kappa \tau+\mu\left(\lambda_{1} \kappa \tau+\lambda_{12}\right)-\sigma_{2} \lambda_{12} \tau\right) z_{1} \\
-\left(\lambda_{2} \lambda_{21}-\nu \lambda_{21}\right) z_{2}-\left(\lambda_{21} \rho_{21} \tau-\lambda_{21} \rho_{12} \kappa \tau+\left(\sigma_{21}+\nu\right) \lambda_{21} \tau\right. \\
\left.\left.+\left(\lambda_{12}-\kappa \tau z_{1}\right)-\left(\lambda_{12} \lambda_{21}-\nu \lambda_{1}\right) z_{21} \kappa \tau\right)\right) \\
d_{12} u=c_{12}+\left(\lambda_{1} \lambda_{12} \varepsilon-\lambda_{1} \rho_{12} \xi+\lambda_{1}\left(\sigma_{21}+\rho_{21}+\nu\right)(1+\xi) \kappa-1\right. \\
\left.-\lambda_{12} \rho_{1} g t-\lambda_{1} \mu(1-\xi)+\lambda_{1} \rho \kappa \tau+\lambda_{12} \rho\right) z_{1} \\
+\left(\lambda_{1} \rho_{2} \kappa^{-1}-\lambda_{21} \rho_{12}-\lambda_{21}^{2}(\alpha+\tau)\right. \\
+\lambda_{21}\left(\sigma_{21}+\nu\right)(1+\xi) \kappa^{-1} \\
\left.-\lambda_{21} \mu(1-\xi)-\lambda_{21}^{2} \tau+\lambda_{2} \rho+\lambda_{21} \rho \kappa \tau\right)\left(z_{12}-\kappa \tau z_{1}\right) \\
d_{21} u=c_{21}+\left(\lambda_{2} \lambda_{12}+\lambda_{2} \rho_{1} \kappa-\lambda_{12} \rho_{12} \kappa+\lambda_{12}\left(\sigma_{12}+\mu\right)(1-\xi) \kappa-\lambda_{12}^{2} \varepsilon \kappa\right. \\
-\lambda_{12} \nu(\xi+1)-\left(\sigma_{12} \kappa+\mu \kappa+\nu\right) \tau \lambda_{1} \kappa \\
+\left(\lambda_{2}^{2}-1+\lambda_{21} \kappa(\alpha+\tau) \lambda_{2}+\lambda_{2} \rho_{12}(1-\xi) \kappa+\lambda_{2} \rho_{21} \xi-\lambda_{12} \rho_{2}\right. \\
\left.+\lambda_{2}\left(\sigma_{12}+\mu\right)(1-\xi) \kappa-\lambda_{2} \nu(\xi+1)+\lambda_{21} \sigma\right) \\
\left.\kappa\left(\sigma_{12} \kappa+\mu \kappa+\nu\right) \lambda_{12}\right) \tau z_{1} \\
+\left(\lambda_{2} \rho_{21}-\lambda_{2} \rho_{12} \kappa-\left(\sigma_{12} \kappa+\mu \kappa+\nu\right)\left(\lambda_{2}+\tau \lambda_{21} \kappa\right)-\lambda_{21} \sigma\right) \tau\left(z_{12}-\kappa \tau z_{1}\right) \\
+\left(\lambda_{2} \lambda_{12}+\lambda_{2} \rho_{1} \kappa-\lambda_{12} \rho_{21}+\lambda_{12}\left(\sigma_{12}+\mu\right)(1-\xi) \kappa\right. \\
\left.-\lambda_{12}^{2} \varepsilon \kappa-\nu \lambda_{12}(\xi+1)+\lambda_{1} \sigma\right) z_{21}
\end{gathered}
$$

one obtains

$$
\begin{gathered}
d_{1 u} \cdot d_{1}=d_{1}, \quad d_{2 u} \cdot d_{2}=d_{2}, \quad d_{1}+d_{2}=u^{-1}, \\
d_{1 u} \cdot d_{12}=d_{12}+\left(\left(\lambda_{21}-\rho \kappa\right) \tau+\mu\right) z_{1}, \\
d_{12 u} \cdot d_{1}=-\left(\left(\lambda_{21}-\rho \kappa\right) \tau+\mu\right) z_{1}, \\
d_{2 u} \cdot d_{21}=d_{21}-\left(\lambda_{2}-\nu\right) z_{2}, \\
d_{21 u} \cdot d_{2}=\left(\lambda_{2}-\nu\right) z_{2}, \\
d_{12 u} \cdot d_{12}=\left(\left(\rho-\lambda_{1} \lambda_{21} \rho_{12}\right)(1-\xi)+\lambda_{21}\left(1-\lambda_{1} \rho_{21}\right)(1+\xi) \kappa^{-1}\right. \\
\left.\quad+\lambda_{1}\left(1-\lambda_{12} \lambda_{21}\right) \varepsilon+\lambda_{21}^{2} \rho_{1}+\lambda_{1}^{2} \rho_{2} \kappa-1-\lambda_{1} \lambda_{21}^{2}(\alpha+\tau)\right) z_{1}, \\
d_{21 u} \cdot d_{21}=\left(\lambda_{2}\left(1-\lambda_{12} \lambda_{21}\right)(\alpha+\tau) \kappa-\left(\sigma_{12} \kappa+\mu \kappa+\nu\right)(1-\xi) \tau \kappa\right. \\
\quad+\sigma(\xi+1)-\lambda_{2} \lambda_{12} \rho_{12}(1-\xi) \kappa-\lambda_{2} \lambda_{12} \rho_{21}(1+\xi) \\
\left.\quad+\lambda_{12}^{2} \rho_{2}+\lambda_{2}^{2} \rho_{1} \kappa-\lambda_{2} \lambda_{12}^{2} \varepsilon \kappa\right) z_{2},
\end{gathered}
$$




$$
\begin{gathered}
d_{12 u} \cdot d_{21}=d_{1}+\left(\lambda_{2} \alpha+\left(\sigma_{12} \kappa+\mu \kappa+\nu\right) \tau(\xi-1)\right. \\
\left.+\sigma(1+\xi) \kappa^{-1}-\sigma_{1}+\nu \tau\right) z_{1} \\
+\left(\rho \kappa \tau-\mu-\lambda_{21} \tau\right)\left(z_{12}-\kappa \tau z_{1}\right)+\left(\nu-\lambda_{2}\right) z_{21}, \\
d_{21 u} \cdot d_{12}=d_{2}+\left(\lambda_{2}-\nu\right) \tau z_{1}-\left(\rho \kappa \tau-\mu-\lambda_{21} \tau\right)\left(z_{12}-\kappa \tau z_{1}\right) \\
-\left(\nu-\lambda_{2}\right) z_{21}+\left(\lambda_{1} \varepsilon \kappa+\lambda_{21}(1+\xi)+\rho(1-\xi) \kappa-\sigma_{2}\right) z_{2},
\end{gathered}
$$

and

$d_{1 u} \cdot z=c_{1} z, \quad d_{2 u} \cdot z=c_{2} z, \quad z_{u} \cdot d_{1}=z c_{1}, \quad z_{u} \cdot d_{2}=z c_{2}$ for all $z \in Z$.

These equations imply that $d_{1}, d_{2}$ form a complete orthogonal system of idempotents of $R^{(u)}$ with Albert (= Peirce)-decomposition given by $R_{11}^{(u)}=$ $k d_{1}+k z_{1}, \quad R_{12}^{(u)}=k d_{12}+k z_{12}, \quad R_{21}^{(u)}=k d_{21}+k z_{21}, \quad R_{22}^{(u)}=k d_{2}+k z_{2}$. The canonical homomorphism $\varphi: R^{(u)} \rightarrow R^{(u)} / Z \cong M_{2}(k)$ maps $d_{1}, d_{2}$ onto an orthogonal system of idempotents of $M_{2}(k)$ and the Albert decomposition of $R^{(u)}$ onto the Peirce decomposition of $M_{2}(k)$. Assume that $R^{(u)}=S^{(u)} \oplus Z$ for some subalgebra $S^{(u)}$ of $R^{(u)}$. Then $S^{(u)} \cong M_{2}(k)$ is associative and contains orthogonal idempotent $s_{1}, s_{2}$ and elements $s_{12}, s_{21}$ such that

$$
\begin{gathered}
s_{1}+s_{2}=u^{-1}, \quad s_{1} s_{12}=s_{12} s_{2}=s_{12}, \quad s_{2} s_{21}=s_{21} s_{1}=s_{21}, \\
s_{12} s_{1}=s_{2} s_{12}=s_{1} s_{21}=s_{21} s_{2}=s_{12}^{2}=s_{21}^{2}=0
\end{gathered}
$$

and $s_{1}-d_{1} \in Z, s_{2}-d_{2} \in Z, s_{12}-d_{12} \in Z, s_{21}-d_{21} \in Z$. Let $z:=s_{1}-d_{1}$ $\in Z$. Then $d_{2}-s_{2}=z$ and the equation $s_{1 u} \cdot s_{1}=d_{1 u} \cdot d_{1}+z_{u} \cdot d_{1}+d_{1 u} \cdot z$ implies $z \in k z_{12}+k z_{21}$. Hence, by suitable choice of $\mu, \nu \in k$ we may assume $d_{1}=s_{1}$ and $d_{2}=s_{2}$. From $s_{12}-d_{12} \in Z \cap R_{12}^{(u)}=k z_{12}$ and $s_{21}-$ $d_{21} \in Z \cap R_{21}^{(u)}=k z_{21}$ we see that by suitable choice of $\rho, \sigma$ we may assume $d_{12}=s_{12}$ and $d_{21}=s_{21}$. Consequently $R^{(u)}$ admits a Wedderburn splitting if and only if there exist $\mu, \nu, \rho, \sigma \in k$ such that the following equations hold

$$
\begin{gathered}
\nu=\lambda_{2}, \quad \mu=\left(\rho \kappa-\lambda_{21}\right) \tau \\
\left(\rho-\lambda_{1} \lambda_{21} \rho_{12}\right)(1-\xi)+\lambda_{21}\left(1-\lambda_{1} \rho_{21}\right)(1+\xi) \kappa^{-1} \\
+\lambda_{1}\left(1-\lambda_{12} \lambda_{21}\right) \varepsilon+\lambda_{21}^{2} \rho_{1}+\lambda_{1}^{2} \rho_{2} \kappa^{-1}-\lambda_{1} \lambda_{21}^{2}(\alpha+\tau)=0 \\
\lambda_{2}\left(1-\lambda_{12} \lambda_{21}\right)(\alpha+\tau)-\left(\sigma_{12} \kappa+\mu \kappa+\lambda_{2}\right)(1-\xi) \tau+\sigma(\xi+1) \kappa^{-1} \\
-\lambda_{2} \lambda_{12} \rho_{12}(1-\xi)-\lambda_{2} \lambda_{12} \rho_{21}(1+\xi) \kappa^{-1} \\
+\lambda_{12}^{2} \rho_{2} \kappa^{-1}+\lambda_{2}^{2} \rho_{1}-\lambda_{2} \lambda_{12}^{2} \varepsilon=0 \\
\lambda_{2} \alpha+\left(\sigma_{12} \kappa+\mu \kappa+\nu\right) \tau(\xi-1)+\sigma(1+\xi) \kappa^{-1}-\sigma_{1}+\nu \tau=0 \\
\lambda_{1} \varepsilon+\lambda_{21}(1+\xi) \kappa^{-1}+\rho(1-\xi)-\sigma_{2} \kappa^{-1}=0 .
\end{gathered}
$$

In this system of linear equations for $\mu, \nu, \rho, \sigma$ the 3 rd and 4 th equations are superfluous as one sees by subtracting the 6th (resp. 5th) equation and inserting the value of $\sigma_{2}$ (resp. $\sigma_{1}$ ). Hence $R^{(u)}$ admits a Wedderburn splitting if and only if the following equations hold 


$$
\begin{aligned}
& \nu=\lambda_{2}, \mu=\left(\rho \kappa-\lambda_{21}\right) \tau, \\
& \rho \kappa(1-\xi)=\sigma_{2}-\lambda_{1} \varepsilon \kappa-\lambda_{21}(1+\xi), \\
& \sigma(1+\xi)=\sigma_{1} \kappa-\lambda_{2}(\alpha+\tau) \kappa-\left(\sigma_{12} \kappa+\mu \kappa+\nu\right) \tau(\xi-1) \kappa .
\end{aligned}
$$

We have proved

THEOREM 3. Let $R=R(\alpha, \varepsilon, \tau, \xi$, $\kappa)$ and

$$
u=\lambda_{1} c_{1}+\lambda_{2} c_{2}+\lambda_{12} c_{12}+\lambda_{21} c_{21}+\rho_{1} z_{1}+\rho_{2} z_{2}+\rho_{12} z_{12}+\rho_{21} z_{21}
$$

with $\lambda_{1} \lambda_{2}-\lambda_{12} \lambda_{21}=1$. Then the isotope $R^{(u)}$ does not admit a Wedderburn splitting if and only if

$$
\xi=1, \quad \lambda_{1} \varepsilon \kappa+2 \lambda_{12} \neq \sigma_{2}=\beta \lambda_{21} \lambda_{1}-\lambda_{21}^{2} \kappa \rho_{1}-\lambda_{1}^{2} \rho_{2}
$$

or

$\xi=-1, \quad \lambda_{2}(\alpha+\tau)-2 \tau\left(\sigma_{12} \kappa+\mu \kappa+\nu\right) \neq \sigma_{1}=\beta \lambda_{12} \lambda_{2}-\lambda_{2}^{2} \rho_{1}-\lambda_{12}^{2} \kappa^{-1} \rho_{2}$, where $\beta=(\alpha+\tau) \lambda_{21}+\varepsilon \lambda_{12}+\rho_{12}(1-\xi)+\rho_{21} \kappa^{-1}(1+\xi)$.

COROLlary 1. For all choices of $\alpha, \varepsilon, \tau, \xi, \kappa(\kappa \neq 0)$ the algebra $R=$ $R(\alpha, \varepsilon, \tau, \xi, \kappa)$ has an isotope $R^{(u)}$ admitting a Wedderburn splitting.

Proof. If $\xi \notin\{1,-1\}$ every isotope $R^{(u)}$ of $R$ admits a Wedderburn splitting. In case $\xi=1 \neq-1$ or $\xi=-1 \neq 1$ the equation $\lambda_{1} \lambda_{2}-\lambda_{12} \lambda_{21}=1$ implies $\lambda_{1} \neq 0$ or $\lambda_{21} \neq 0$ and $\lambda_{2} \neq 0$ or $\lambda_{12} \neq 0$. Hence one can choose $\rho_{1}, \rho_{2}$ so that

$$
\begin{aligned}
& \lambda_{1}^{2} \rho_{2}+\lambda_{21}^{2} \kappa \rho_{1}=\beta \lambda_{21} \lambda_{1}-\lambda_{1} \varepsilon \kappa-2 \lambda_{12} \text { resp. } \\
& \lambda_{12}^{2} \kappa^{-1} \rho_{2}+\lambda_{2}^{2} \rho_{1}=\beta \lambda_{12} \lambda_{2}-\lambda_{2}(\alpha+\tau)+2 \tau\left(\sigma_{12} \kappa+\mu \kappa+\nu\right)
\end{aligned}
$$

and $R^{(u)}$ admits a Wedderburn splitting. In the remaining case $\xi=1=-1$ both equations have a common solution $\rho_{1}, \rho_{2}$ since the determinant is $\lambda_{1}^{2} \lambda_{2}^{2}-\lambda_{12}^{2} \lambda_{21}^{2}=\left(\lambda_{1} \lambda_{2}-\lambda_{12} \lambda_{21}\right)^{2}=1$.

COROLlaRY 2. For all choices of $\alpha, \varepsilon, \tau, \xi, \kappa(\kappa \neq 0)$ the quadratic Jordan algebra $R^{+}=R(\alpha, \varepsilon, \tau, \xi, \kappa)^{+}$has an isotope $\left(R^{+}\right)^{(u)}$ admitting a Wedderburn splitting.

Proof. A Wedderburn splitting of $R^{(u)}$ induces a Wedderburn splitting of $\left(R^{(u)}\right)+=\left(R^{+}\right)^{(u)}$.

Noncommutative Jordan algebras. The (left) homotopes of $A^{(u)}$ of a noncommutative Jordan algebra $A$ are again noncommutative Jordan algebras [3]. For unital $A$ and invertible $u \in A$ we call $A^{(u)}$ the $u$-isotope. Let $A$ be the noncommutative Jordan algebra over a field $k$ of characteristic $\neq 2$ with unit element $e$ and basis $e, x, y, z$ whose multiplication is determined by $x^{2}=y^{2}=e, x y=z=-y x, x z=z x=y z=z y=z^{2}=0$ [11]. Here one can show

THEOREM 4. $A$ is a noncommutative Jordan algebra such that no isotope $A^{(u)}$ of $A$ admits $a$ Wedderburn splitting.

Proof. Let $u=\rho e+\alpha x+\beta y+\gamma z \in A$. The equation $u^{2}=\left(\alpha^{2}+\beta^{2}-\right.$ 
$\left.\rho^{2}\right) e+2 \rho u$ implies that $u$ is invertible if and only if $\alpha^{2}+\beta^{2}-\rho^{2} \neq 0$. The multiplication of $a, b \in A$ in $A^{(u)}$ is given by $a_{u} \cdot b:=(a u) b+(u b) a-u(b a)$. Hence the basis $e, x, y, z$ of $A^{(u)}$ satisfies the multiplication table

\begin{tabular}{c|cccc} 
& $e$ & $x$ & $y$ & $z$ \\
\hline$e$ & $\rho e+\alpha x+\beta y+\gamma z$ & $\alpha e+\rho x-\beta z$ & $\beta e+\rho y+\alpha z$ & $\rho z$ \\
$x$ & $\alpha e+\rho x+\beta z$ & $\rho e+\alpha x-\beta y-\gamma z$ & $\beta x+\alpha y+\rho z$ & $\alpha z$ \\
$y$ & $\beta e+\rho y-\alpha z$ & $\beta x+\alpha y-\rho z$ & $\rho e-\alpha x+\beta y-\gamma z$ & $\beta z$ \\
$z$ & $\rho z$ & $\alpha z$ & $\beta z$ & 0
\end{tabular}

The radical is $Z=k z$. If $A^{(u)}$ admits a Wedderburn splitting, then there exist $\xi, \eta, \varepsilon \in k$ such that $x+\xi z, y+\eta z, e+\varepsilon z$ multiply in the same way as $x+Z, y+Z, e+Z$ in $A / Z$. The commutator in $A^{(u)}$ of $a, b$ is $[a, b]^{(u)}=$ $a_{u} \cdot b-b_{u} \cdot a=[u, b] a+[a, u] b+u[a, b]$. Hence $[x+\xi z, y+\eta z]^{(u)}=$ $2 \rho z,[x+\xi z, e+\varepsilon z]^{(u)}=2 \beta z,[e+\varepsilon z, y+\eta z]^{(u)}=2 \alpha z$. There is no choice of $\xi, \eta, \varepsilon$ such that $2 \rho z=2 \beta z=2 \alpha z=0$ i.e., $\alpha=\beta=\rho=0$ since $u$ is invertible. Hence $A^{(u)}$ does not admit a Wedderburn splitting.

ADDED IN PROOF. By letter of January 20, 1978, I. P. Shestakov informed the author that he independently constructed an eight-dimensional right alternative algebra that does not permit a Wedderburn decomposition.

\section{REFERENCES}

1. R. Carlsson, Der Wedderburnsche Hauptsatz für alternative Tripelsysteme und Paare, Math. Ann. 228 (1977), 233-248.

2. N. Jacobson, Structure and representation of Jordan algebras, Amer. Math. Soc. Colloq. Publ., vol. 39, Amer. Math. Soc., Providence, R. I., 1968.

3. K. McCrimmon, Homotopes of noncommutative Jordan algebras, Math. Ann. 191 (1971), 263-270.

4. I. M. Mikheev, Wedderburn's theorem on detachment of the radical for $(-1,1)$-algebras, Algebra and Logic 12 (1973), 168-172.

5. A. A. Nikitin, Almost alternative algebras, Algebra and Logic 13 (1974), 287-305.

6. D. J. Rodabaugh, On the Wedderburn principal theorem, Trans. Amer. Math. Soc. 138 (1969), 343-361.

7. R. D. Schafer, Standard algebras, Pacific J. Math. 29 (1969), 203-223.

8. F. P. Shestakov, Generalized standard rings, Algebra and Logic 13 (1974), 46-56.

9. H. F. Smith, The Wedderburn principal theorem for generalized alternative algebras I, Trans. Amer. Math. Soc. 212 (1975), 139-148.

10. __ Paper on locally $(1,-1)$-algebras (in preparation).

11. A. Thedy, Zum Wedderburnschen Zerlegungssatz, Math. Z. 113 (1970), 173-195.

12. ___ Right alternative rings, J. Algebra 37 (1975), 1-43.

13. __ Right alternative rings with Peirce decomposition, J. Algebra 37 (1975), 44-63.

14. —_, Nil-semisimple right alternative algebras, J. Algebra 48 (1977), 390-400.

15. __, Right alternative rings with minimal condition, Math. Z. 155 (1977), 277-286.

Abtenlung Mathematix, Universität Dortmund, 4600 Dortmund, Federal Republic of Germany 